\title{
THE FIRST DETECTIONS OF THE KEY PREBIOTIC MOLECULE PO IN STAR-FORMING REGIONS
}

\author{
V. M. Rivilla ${ }^{1}$, F. Fontani ${ }^{1}$, M. T. Beltrán ${ }^{1}$, A. Vasyunin ${ }^{2,3}$, P. Caselli ${ }^{2}$, J. Martín-Pintado $^{4}$, and R. Cesaroni ${ }^{1}$ \\ ${ }^{1}$ Osservatorio Astrofisico di Arcetri, Largo Enrico Fermi 5, I-50125, Firenze, Italy \\ ${ }^{2}$ Max-Planck Institute for Extraterrestrial Physics, Giessenbachstrasse 1, D-85748, Garching, Germany \\ ${ }^{3}$ Ural Federal University, Ekaterinburg, Russia \\ ${ }^{4}$ Centro de Astrobiología (CSIC-INTA), Ctra. de Torrejón a Ajalvir km 4, E-28850, Torrejón de Ardoz, Madrid, Spain \\ Received 2016 April 28; revised 2016 May 14; accepted 2016 May 17; published 2016 July 28
}

\begin{abstract}
Phosphorus is a crucial element in biochemistry, in particular the $\mathrm{P}-\mathrm{O}$ bond, which is key in the formation of the backbone of deoxyribonucleic acid. So far, PO has only been detected toward the envelope of evolved stars, but never toward star-forming regions. We report the first detection of PO toward two massive star-forming regions, W51 e1/e2 and $\mathrm{W} 3(\mathrm{OH})$, using data from the IRAM $30 \mathrm{~m}$ telescope. PN has also been detected toward the two regions. The abundance ratio $\mathrm{PO} / \mathrm{PN}$ is 1.8 and 3 for $\mathrm{W} 51$ and $\mathrm{W} 3(\mathrm{OH})$, respectively. Our chemical model indicates that the two molecules are chemically related and are formed via gas-phase ion-molecule and neutralneutral reactions during cold collapse. The molecules freeze out onto grains at the end of the collapse and desorb during the warm-up phase once the temperature reaches $\sim 35 \mathrm{~K}$. Similar abundances of the two species are expected during a period of $\sim 5 \times 10^{4} \mathrm{yr}$ at the early stages of the warm-up phase, when the temperature is in the range $35-90 \mathrm{~K}$. The observed molecular abundances of $10^{-10}$ are predicted by the model if a relatively high initial abundance of $5 \times 10^{-9}$ of depleted phosphorus is assumed.
\end{abstract}

Key words: astrobiology - astrochemistry - ISM: molecules - line: identification - molecular data stars: formation

\section{INTRODUCTION}

The detection of new interstellar molecules related to prebiotic chemistry in star-forming regions will allow us to make progress in understanding how the building blocks of life could originate in the interstellar medium (ISM). However, a key ingredient still evades detection: phosphorus, P. This element is essential for life (Maciá et al. 1997), because it plays a central role in the formation of P-bearing macromolecules such as phospholipids, which are the structural components of all cellular membranes. Moreover, living cells on Earth use P-bearing compounds, phosphates, to transport energy in the form of adenosine triphosphate (see, e.g., Pasek \& Lauretta 2005). Particularly important to basic biochemistry is the $P$ $-\mathrm{O}$ bond, which is fundamental for many relevant biological molecules such as phosphate esters, which are essential for the formation of the backbone of the genetic macromolecule deoxyribonucleic acid.

Phosphorus is thought to be synthesized in massive stars and injected to the ISM through supernova explosions (Koo et al. 2013). It is a cosmically relatively abundant element, $\mathrm{P} / \mathrm{H} \sim 3 \times 10^{-7}$ (Grevesse \& Sauval 1998). It has been detected toward the atmospheres of stars (Caffau et al. 2011, 2016; Roederer et al. 2014), but it is barely detected in the ISM. The ion $\mathrm{P}^{+}$has been detected in several diffuse clouds (Jura \& York 1978) and only a few simple P-bearing species (PN, PO, $\mathrm{CP}, \mathrm{HCP}, \mathrm{C}_{2} \mathrm{P}, \mathrm{PH}_{3}$ ) have been identified toward the circumstellar envelopes of very evolved objects (Tenenbaum et al. 2007; De Beck et al. 2013; Agúndez et al. 2014). In starforming regions, only PN has been detected so far (Turner \& Bally 1987; Ziurys \& Friberg 1987; Fontani et al. 2016). Previous searches for PO toward star-forming regions (Sutton et al. 1985; Matthews et al. 1987) have been unsuccessful.

Despite the astrobiological importance of P-bearing molecules, their chemistry is still poorly understood in the ISM. The few theoretical models devoted to P-chemistry disagree in both the chemical formation pathways and the predictions of the abundances of PO and PN. While some works (Millar et al. 1987; Adams et al. 1990; Charnley \& Millar 1994) suggest that PN should be more abundant than PO, other studies involving theoretical modeling and laboratory experiments (e.g., Thorne et al. 1984) predict that P should be found mainly in the form of PO.

To constrain the chemical models and understand the chemistry of phosphorus in the ISM, astronomical detections of $\mathrm{PO}$ and $\mathrm{PN}$ in star-forming regions are required. We present observations searching for $\mathrm{PN}$ and $\mathrm{PO}$ toward two massive starforming regions: the $\mathrm{W} 51 \mathrm{e} 1 / \mathrm{e} 2$ and the $\mathrm{W} 3(\mathrm{OH})$ complexes (with luminosities of $1.5 \times 10^{6}$ and $1.0 \times 10^{5} L_{\odot}$, respectively). The W51 star-forming region, located at a distance of $5.1 \mathrm{kpc}$ (Xu et al. 2009) in the Sagittarius arm of our Galaxy, harbors two compact radio sources (Scott 1978) known as e1 and e2, which are associated with hot molecular cores with a rich chemistry (Zhang \& Ho 1997; Zhang et al. 1998; Ikeda et al. 2001; Liu et al. 2001; Remijan et al. 2002; Demyk et al. 2008; Kalenskii \& Johansson 2010; Lykke et al. 2015). The W3(OH) complex, located at $2.04 \mathrm{kpc}$ (Hachisuka et al. 2006), harbors two massive objects separated by $6^{\prime \prime}$ : the UC HII region $\mathrm{W} 3(\mathrm{OH})$ (Wilner et al. 1995) and the chemically rich $\mathrm{W} 3\left(\mathrm{H}_{2} \mathrm{O}\right)$ hot molecular core (Wyrowski et al. 1999; Zapata et al. 2011; Qin et al. 2015).

In this paper, we report the first detection of PO toward these two massive star-forming regions.

\section{OBSERVATIONS}

We used data from different observing runs performed with the IRAM $30 \mathrm{~m}$ telescope at Pico Veleta (Spain) in 2012 and $2015 .{ }^{5}$ The dates, positions, and frequency coverages of these observations are summarized in Table 1. The W51 e1/e2

The 2012 December data were obtained from Lykke et al. (2015). 
Table 1

Summary of the IRAM 30 m Observations Toward the W51 and W3(OH) Hot Molecular Cores

\begin{tabular}{|c|c|c|c|c|}
\hline Date & Source & R.A.J2000 & decl.J2000 $_{\text {J20 }}$ & Frequency Coverage $(\mathrm{GHz})$ \\
\hline 2012 Apr 25 & $\begin{array}{c}\text { W51 } \\
\text { W3(OH) }\end{array}$ & $\begin{array}{c}19^{\mathrm{h}} 23^{\mathrm{m}} 43^{\mathrm{s}} \cdot 90 \\
2^{\mathrm{h}} 27^{\mathrm{m}} 04^{\mathrm{s}} .69\end{array}$ & $\begin{array}{rrr}14^{\circ} & 30^{\prime} & 32 ! \prime 0 \\
61^{\circ} & 52^{\prime} & 25^{\prime \prime} 51\end{array}$ & $\begin{array}{c}88.83-96.60 \text { and } \\
104.51-112.29\end{array}$ \\
\hline $2015 \operatorname{Dec} 9$ & W51 & $19^{\mathrm{h}} 23^{\mathrm{m}} 43^{\mathrm{s}} .90$ & $14^{\circ} 30^{\prime} 32^{\prime \prime} 0$ & $\begin{array}{c}134.47-142.25 \text { and } \\
150.15-157.90\end{array}$ \\
\hline
\end{tabular}

(hereafter W51) region was observed at 1,2 , and $3 \mathrm{~mm}$, while the $\mathrm{W} 3(\mathrm{OH})$ region was observed at $3 \mathrm{~mm}$.

All observing campaigns used the Eight Mixer Receiver and the Fast Fourier Transform Spectrometer, which provides a spectral resolution of $0.195 \mathrm{kHz}$. The spectra were exported from the software package CLASS of GILDAS ${ }^{6}$ to MADCUBAIJ ${ }^{7}$ which was used for line identification and analysis. The line intensities of the IRAM $30 \mathrm{~m}$ spectra were converted to the main-beam temperature $T_{\mathrm{mb}}$ scale, using the efficiencies provided by IRAM $^{8}$. The half-power beam widths of the observations can be estimated using the expression $\theta_{\text {beam }}[\operatorname{arcsec}]=2460 / \nu(\mathrm{GHz})$.

\section{RESULTS}

\subsection{Detection of $P N$}

We detected the $J=2-1$ and $J=3-2$ transitions of PN (Table 2) toward W51 (the left and middle panels of Figure 1). This molecule was already detected toward this source by Turner \& Bally (1987) using the NRAO $12 \mathrm{~m}$ telescope. The comparison of the line intensities of the PN $J=2-1$ transition observed with the 30 and $12 \mathrm{~m}$ telescopes toward W51 allows us to estimate the size of the PN emission. The approximate size of the emitting region, $\theta_{\mathrm{s}}$, can be estimated from the following expression:

$$
T_{12 \mathrm{~m}} \frac{\theta_{\mathrm{s}}^{2}+\theta_{12 \mathrm{~m}}^{2}}{\theta_{\mathrm{s}}^{2}}=T_{30 \mathrm{~m}} \frac{\theta_{\mathrm{s}}^{2}+\theta_{30 \mathrm{~m}}^{2}}{\theta_{\mathrm{s}}^{2}},
$$

where $\theta_{12 \mathrm{~m}}=69^{\prime \prime}$ and $\theta_{30 \mathrm{~m}}=26^{\prime \prime}$ are the half-power beam widths of the two telescopes, and $T_{12 \mathrm{~m}}=20 \mathrm{mK}$ and $T_{30 \mathrm{~m}}=120 \mathrm{mK}$ are the corresponding main-beam brightness temperatures. We obtain an angular size of the emitting region of $\sim 12^{\prime \prime}$. Using this angular diameter, the LTE simulated spectra reproduce the PN $J=2-1$ and $J=3-2$ transitions for an excitation temperature of $\sim 35 \mathrm{~K}$ (Figure 1), as previously found by Turner \& Bally (1987). We derived a PN sourceaveraged column density of $2.1 \times 10^{13} \mathrm{~cm}^{-2}$ (Table 3 ). Using the derived $\mathrm{H}_{2}$ column density $N_{\mathrm{H}_{2}}$ (see the details of the calculation in the Appendix), the molecular abundance with respect to molecular hydrogen is $1.1 \times 10^{-10}$.

We have also detected the $J=2-1$ PN transition toward $\mathrm{W} 3(\mathrm{OH})$ (right panel of Figure 1). To our knowledge this is the first detection of PN toward this source. Assuming the same

\footnotetext{
6 The GILDAS software is available at http://www.iram.fr/IRAMFR/ GILDAS.

7 Madrid Data Cube Analysis on ImageJ is a software package developed in the Center of Astrobiology (Madrid, INTA-CSIC) to visualize and analyze single spectra and datacubes (Martín-Pintado et al. 2016).

8 http://www.iram.es/IRAMES/mainWiki/Iram30mEfficiencies
}

Table 2

Spectroscopic Parameters of Targeted PN Transitions, from CDMS

\begin{tabular}{lccr}
\hline \hline Frequency $(\mathrm{GHz})$ & Transition & $S_{i j} \mu^{2}\left(\mathrm{D}^{2}\right)$ & $E_{\text {up }}(\mathrm{K})$ \\
\hline 93.97977 & $2-1$ & 15.1 & 6.8 \\
140.96769 & $3-2$ & 22.6 & 13.5 \\
\hline
\end{tabular}

excitation temperature and linear diameter as that of W51 (i.e., $30^{\prime \prime}$ at the distance of $\left.\mathrm{W} 3(\mathrm{OH})\right)$, we obtained a source-averaged PN column density of $0.2 \times 10^{13} \mathrm{~cm}^{-2}$, which implies a molecular abundance of $0.4 \times 10^{-10}$.

\subsection{Detection of $P O$}

The PO radical is a diatomic molecule with a dipole moment of $\mu=1.88 \mathrm{D}$ (Kanata et al. 1988). Its millimeter spectrum was measured in the gas phase in the laboratory by Kawaguchi et al. (1983). PO has a ${ }^{2} \Pi_{\mathrm{r}}$ ground electronic state, and therefore each $J$-transition splits into a doublet because of $\Lambda$ coupling. The spin of $I=1 / 2$ of the phosphorus atom produces a further splitting of each $\Lambda$ doublet into two hyperfine levels. Table 4 shows the PO transitions that are most likely detectable in the ISM with the $30 \mathrm{~m}$ telescope: the brightest transitions of the $\Omega=1 / 2$ ladder, with $S_{i j} \mu^{2}>5 \mathrm{D}^{2}$, and with energy levels $E_{\mathrm{up}}<300 \mathrm{~K}$. The lower energy $\left(E_{\mathrm{up}}=3 \mathrm{~K}\right)$ and lower frequency transitions $(J=3$ / $2-1 / 2$ ) at $65 \mathrm{GHz}$ of the $\Omega=1 / 2$ ladder are not observable due to atmospheric opacity. The $J=9 / 2-7 / 2$ quadruplet falls at $196 \mathrm{GHz}$, outside the range covered by the IRAM $30 \mathrm{~m}$ telescope. The search for higher energy PO transitions (e.g., $J=13 / 2-11 / 2$ at $283 \mathrm{GHz}$ ) is much less feasible, due to strong contamination from brighter molecular lines at this frequency. Therefore, the PO transitions given in Table 4 are the best candidates for a detection. The observations toward W51 covered all these PO transitions (at $1,2$, and $3 \mathrm{~mm})$, while the observations toward $\mathrm{W} 3(\mathrm{OH})$ only covered the $3 \mathrm{~mm}$ transitions.

We have used MADCUBAIJ to identify the PO transitions in the observed spectra. We have assumed the same temperature $\left(T_{\mathrm{ex}}=35 \mathrm{~K}\right)$ and source size as that of PN to produce the LTE spectrum. We show the $3 \mathrm{~mm}$ spectra of W51 and the LTE fit in Figure 2. We have clearly identified the PO quadruplet toward W51. The four transitions are well fitted by the LTE model. The lines are detected above $6 \sigma$. We note that these PO transitions have already been detected in the wind of the oxygen-rich AGB star IK Tauri by De Beck et al. (2013).

The PO transitions at $108.99845 \mathrm{GHz}$ and $109.04540 \mathrm{GHz}$ are completely free of contamination. The transition at 109.20620 GHz also seems unblended, although the LTE model slightly underestimates the observed intensities. The transition at 109.28119 GHz appears clearly blended with another species. To firmly confirm the detection of PO, we have evaluated the 

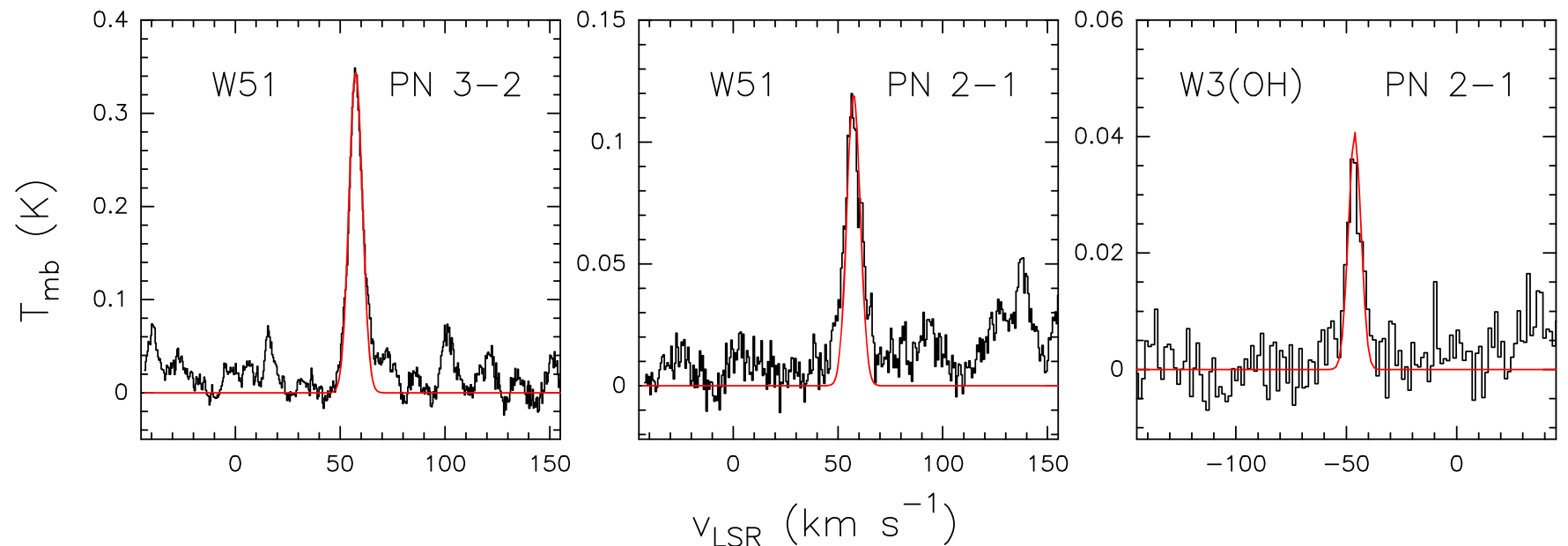

Figure 1. PN transitions observed toward W51 and W3(OH). The red line is the LTE fit for a excitation temperature of $35 \mathrm{~K}$ (see text) and the source-average column densities given in Table 3.

Table 3

Physical Parameters of PO and PN from the LTE Analysis

\begin{tabular}{|c|c|c|c|c|c|c|c|}
\hline Molecule & Source & $\begin{array}{l}T_{\mathrm{ex}} \\
(\mathrm{K})\end{array}$ & $N_{\mathrm{s}}$ & $\left(\times 10^{13} \mathrm{~cm}^{-2}\right)^{N_{\mathrm{b}}}$ & $\begin{array}{c}v_{\mathrm{LSR}} \\
\left(\mathrm{km} \mathrm{s}^{-1}\right)\end{array}$ & $\begin{array}{c}\Delta v \\
\left(\mathrm{~km} \mathrm{~s}^{-1}\right)\end{array}$ & $\begin{array}{c}X_{\mathrm{s}} \\
\left(10^{-10}\right)\end{array}$ \\
\hline \multirow[t]{2}{*}{ PN } & W51 & 35 & 2.1 & 0.4 & 57.5 & 8.2 & 1.1 \\
\hline & $\mathrm{W} 3(\mathrm{OH})$ & 35 & 0.2 & 0.13 & -46.2 & 6.6 & 0.4 \\
\hline \multirow[t]{2}{*}{$\mathrm{PO}$} & W51 & 35 & 4.0 & 0.7 & 57.0 & 7.0 & 2.0 \\
\hline & W3(OH) & 35 & 0.6 & 0.3 & -46.2 & 6.6 & 1.2 \\
\hline
\end{tabular}

Note. $T_{\text {ex }}$ (excitation temperature), $N_{\mathrm{s}}$ (source-averaged column density), $N_{\mathrm{b}}$ (beam-averaged column density), $v_{\mathrm{LSR}}$ (central velocity of the molecular emission), $\Delta v$ (molecular linewidth, i.e., the FWHM of the line Gaussian profile), and $X_{\mathrm{s}}$ (source-averaged molecular abundances).

Table 4

Spectroscopic Parameters of Targeted PO Transitions $\left(S_{i j} \mu^{2}>5 \mathrm{D}^{2}\right.$ and $\left.E_{\text {up }}(\mathrm{K})<300 \mathrm{~K}\right)$, from CDMS

\begin{tabular}{|c|c|c|c|}
\hline Frequency $(\mathrm{GHz})$ & Transition & $S_{i j} \mu^{2}\left(\mathrm{D}^{2}\right)$ & $E_{\text {up }}(\mathrm{K})$ \\
\hline \multicolumn{4}{|c|}{$J=5 / 2-3 / 2, \Omega=1 / 2$} \\
\hline 108.99845 & $F=3-2, l=e$ & 9.9 & 8.4 \\
\hline 109.04540 & $F=2-1, l=e$ & 6.4 & 8.4 \\
\hline 109.20620 & $F=3-2, l=f$ & 9.9 & 8.4 \\
\hline 109.28119 & $F=2-1, l=f$ & 6.4 & 8.4 \\
\hline \multicolumn{4}{|c|}{$J=7 / 2-5 / 2, \Omega=1 / 2$} \\
\hline 152.65698 & $F=4-3, l=e$ & 13.6 & 15.7 \\
\hline 152.68028 & $F=3-2, l=e$ & 10.1 & 15.7 \\
\hline 152.85545 & $F=4-3, l=f$ & 13.6 & 15.7 \\
\hline 152.88813 & $F=3-2, l=f$ & 10.1 & 15.7 \\
\hline \multicolumn{4}{|c|}{$J=11 / 2-9 / 2, \Omega=1 / 2$} \\
\hline 239.94898 & $F=6-5, l=e$ & 20.9 & 36.7 \\
\hline 239.95810 & $F=5-4, l=e$ & 17.4 & 36.7 \\
\hline 240.14106 & $F=6-5, l=f$ & 20.9 & 36.7 \\
\hline 240.15253 & $F=5-4, l=f$ & 17.4 & 36.7 \\
\hline
\end{tabular}

possibility of contamination from lines of other molecules. We have searched in the available catalogs $\left(\mathrm{CDMS}^{9}\right.$ and $\left.\mathrm{JPL}^{10}\right)$ and conclude that there are no transitions blended with the PO lines at 108.99845 and $109.04540 \mathrm{GHz}$. The only possible contamination

\footnotetext{
9 http://www.astro.uni-koeln.de/cdms

10 http://spec.jpl.nasa.gov/
}

of the PO transition at $109.20620 \mathrm{GHz}$ is the complex organic molecule ethyl formate, $\mathrm{C}_{2} \mathrm{H}_{5} \mathrm{OCHO}$. Interestingly, this molecule has been recently identified toward W51 (Rivilla et al. 2016) based on the detection of multiple transitions, which has allowed us to derive its rotational temperature and column density. Using the physical parameters derived in Rivilla et al. (2016) $\left(N=10^{16.3}, T=100 \mathrm{~K}\right)$, we have predicted the LTE line profile of the ethyl formate transition close to the $109.20620 \mathrm{GHz}$ transition of PO. The result is shown in the left panel of Figure 3. Ethyl formate has a very weak contribution to the observed emission, which is hence mainly due to PO. The LTE profile reproduces very well the observed line profile if the emission of both molecules is taken into account (see the left panel of Figure 3).

In the case of the $109.28119 \mathrm{GHz}$ transition of PO, we have found that it is blended with a line of ethylene glycol, $\left(\mathrm{CH}_{2} \mathrm{OH}\right)_{2}$. This species has been tentatively identified toward W51 by Lykke et al. (2015) from observations at $1 \mathrm{~mm}$. Our 2 and $3 \mathrm{~mm}$ spectra clearly confirm the detection of ethylene glycol, and allow us to confirm the excitation temperature of $\sim 120 \mathrm{~K}$ previously obtained by Lykke et al. (2015). We show in the right panel of Figure 3 the LTE model of the ethylene glycol transition close to the $109.28119 \mathrm{GHz}$ transition of PO. The combined contribution of PO and ethylene glycol reproduces well the observed spectral line profile.

The LTE fit of the four PO spectral features at $3 \mathrm{~mm}$ gives a source-averaged PO column density of $4 \times 10^{13} \mathrm{~cm}^{-2}$ and a molecular abundance of $2 \times 10^{-10}$ (Table 3). In Figure 4 we also show the predicted LTE lines profile of the PO at 1 and $2 \mathrm{~mm}$ (Table 4). The LTE prediction is consistent with the 


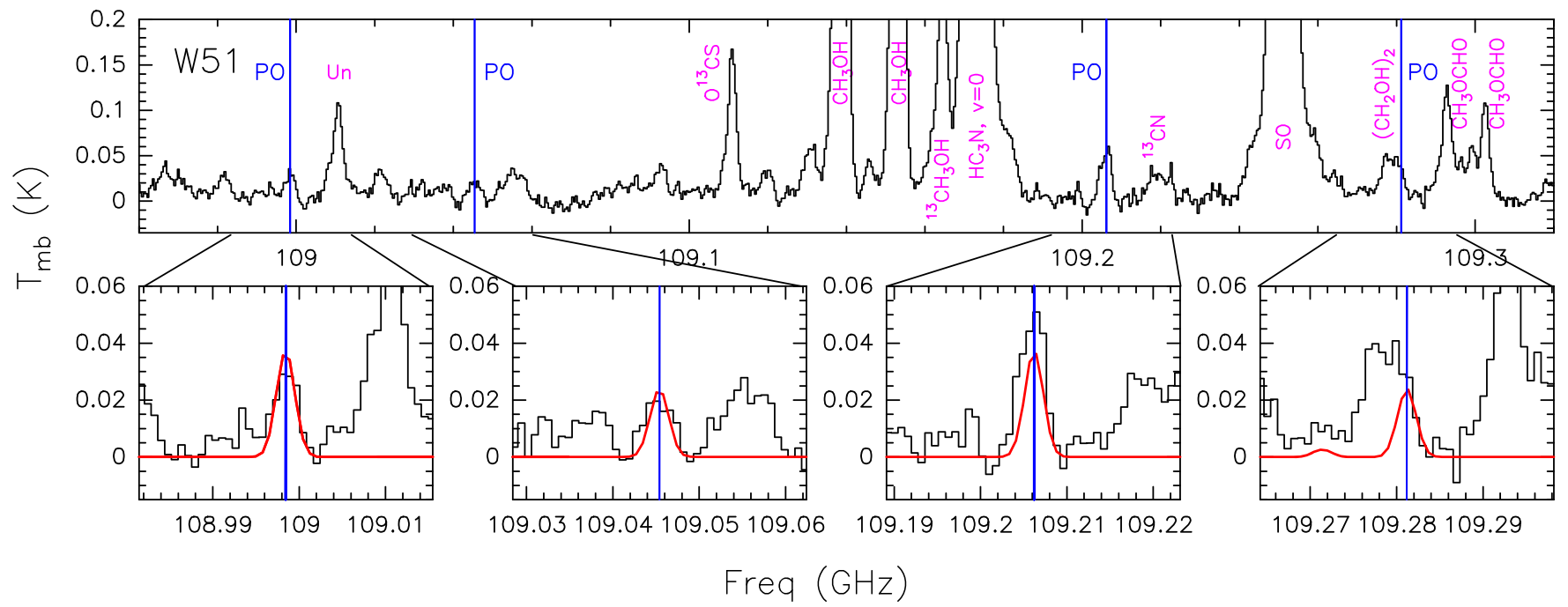

Figure 2. Spectrum observed at $3 \mathrm{~mm}$ toward W51. The PO transitions are indicated with blue vertical lines. The lower panels show zoom-in views of the PO transitions. The red line is the LTE fit with a excitation temperature of $35 \mathrm{~K}$ and an assumed source size of $12^{\prime \prime}$ (see text).
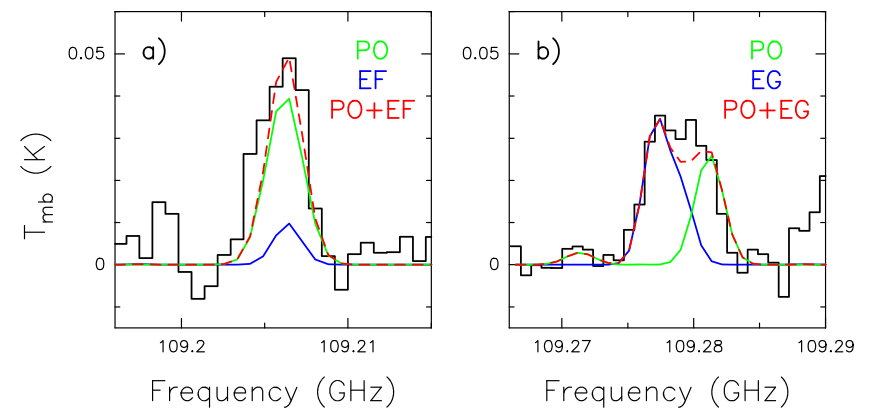

Figure 3. Simultaneous LTE fit of the PO transitions at 109.206 and $109.281 \mathrm{GHz}$ and transitions of ethyl formate (EF) and ethylene glycol (EG) that are partially blended.

observed spectra. The predicted PO transition at $240.14106 \mathrm{GHz}$ fits a partially blended line of the observed spectrum (Figure 4), while the other PO transitions appear heavily blended with brighter lines of other molecular species. In summary, we have detected all 12 detectable PO transitions toward W51 with intensities consistent with LTE emission: two of them (at $3 \mathrm{~mm}$ ) are unblended, two (at $3 \mathrm{~mm}$ ) are partially blended, while the others (all the 2 and $1 \mathrm{~mm}$ transitions) are heavily blended.

For $\mathrm{W} 3(\mathrm{OH})$ we show in Figure 5 the observed $3 \mathrm{~mm}$ spectra. The two brightest lines of PO $(108.99845 \mathrm{GHz}$ and $109.20620 \mathrm{GHz}$ ) are detected at a $\sim 4 \sigma$ level. We have fitted these lines with the LTE model, assuming $T_{\mathrm{ex}}=35 \mathrm{~K}$ and the same diameter assumed for PN (30) density of $0.6 \times 10^{13} \mathrm{~cm}^{-2}$ and a molecular abundance of $1.2 \times 10^{-10}$ (Table 3). To confirm this detection we have stacked the $3 \mathrm{~mm}$ observed spectra to improve the signal to noise ratio. This method is used to identify molecular species marginally detected in several lines. In practice, we have averaged the observed spectra of the four PO transitions with the weights calculated assuming LTE, and normalized the result to the peak value. The resulting spectrum, shown in Figure 6 , indicates a clear detection at the systemic velocity of the source. The signal is $>5 \sigma$, confirming the detection of $\mathrm{PO}$ toward $\mathrm{W} 3(\mathrm{OH})$.

\section{DISCUSSION: THE CHEMISTRY OF PHOSPHORUS}

\subsection{The PO/PN Ratio}

The LTE fits of PO and PN transitions show that the molecular abundances of the two molecules are similar in the two sources $\left(\sim 10^{-10}\right)$, the PO abundance being higher by a factor $\sim 2$. Our detections toward W51 and W3 $(\mathrm{OH})$ show that the line intensities of $\mathrm{PO}$ at $3 \mathrm{~mm}$ are a factor $\geqslant 4$ weaker than the PN $J=2-1$ transition at similar wavelengths. This makes the identification of PO more difficult than that of PN and explains why previous attempts to detect PO were fruitless. Matthews et al. (1987) derived upper limits of the beam averaged column densities ${ }^{11}$ of four hot molecular cores of $<(0.2-2.7) \times 10^{13} \mathrm{~cm}^{-2}$. These upper limits are consistent with the PO column densities we have derived, suggesting that the non-detections of Matthews et al. (1987) were due to insufficient sensitivity.

More recently, Fontani et al. (2016) have found upper limits for the $\mathrm{PO} / \mathrm{PN}$ ratio of $<(1.3-4.5)$ toward a sample of massive star-forming cores (see Table 5), which are also consistent with the abundances of both P-bearing species estimated by us. The upper limit of the $\mathrm{PO} / \mathrm{PN}$ ratio of $\sim 0.5-1.3$ found toward a protostellar shock by Aota \& Aikawa (2012) is also in agreement with our observed ratios.

Before the present detections, PO had only been detected toward the envelopes of two evolved stars, VY Canis Majoris (Tenenbaum et al. 2007), and IK Tauri (De Beck et al. 2013), where the $\mathrm{PO} / \mathrm{PN}$ ratios are 2.2 and $0.17-2$, respectively. These ratios are similar to those found toward W51 and $\mathrm{W} 3(\mathrm{OH})$, suggesting that phosphorus seems to be equally distributed in the form of $\mathrm{PO}$ and $\mathrm{PN}$ in both interstellar and circumstellar material.

\subsection{Chemical Model: Constraining the Phosphorus Chemistry}

We have investigated the formation of the phosphorusbearing molecules $\mathrm{PO}$ and $\mathrm{PN}$ in massive star-forming regions

\footnotetext{
${ }^{11}$ Matthews et al. (1987) derived the PO upper limits assuming a dipole moment of PO of 2.13 Debye. Kanata et al. (1988) measured a dipole moment of 1.88 , so we have corrected the PO upper limits of Table 1 of Matthews et al. (1987) by multiplying for a factor $(2.13 / 1.88)^{2} \simeq 1.3$.
} 

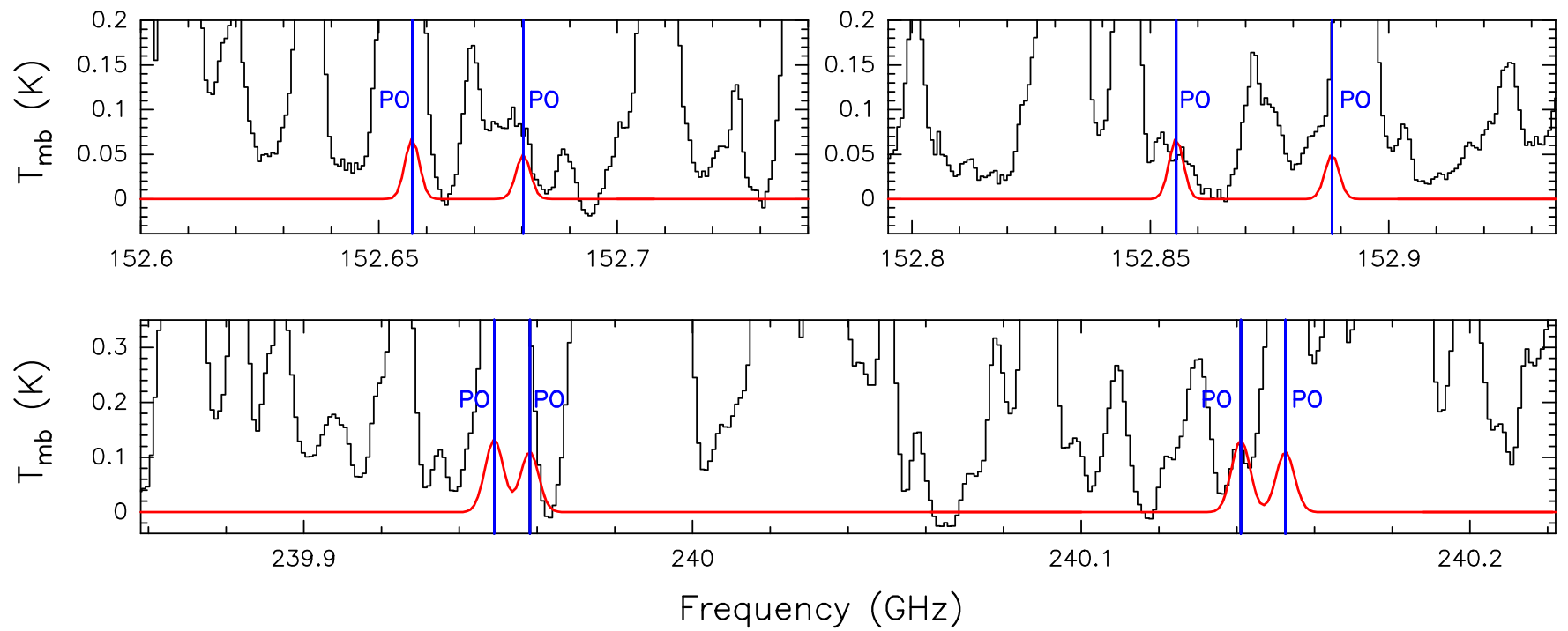

Figure 4. PO transitions at 1 and $2 \mathrm{~mm}$ observed toward the W51 hot core. The PO transitions are indicated with blue vertical lines and the red line is the LTE fit as in Figure 2.

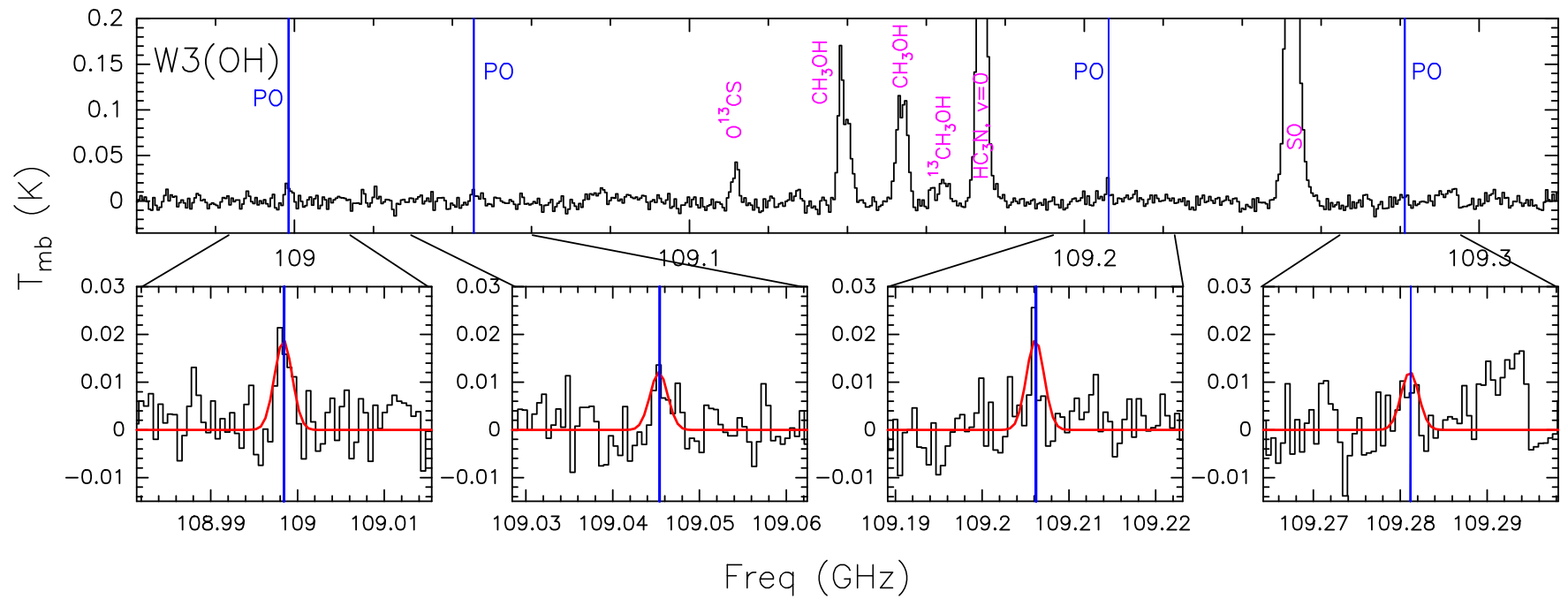

Figure 5. Same as Figure 2 for the W3(OH) hot core.

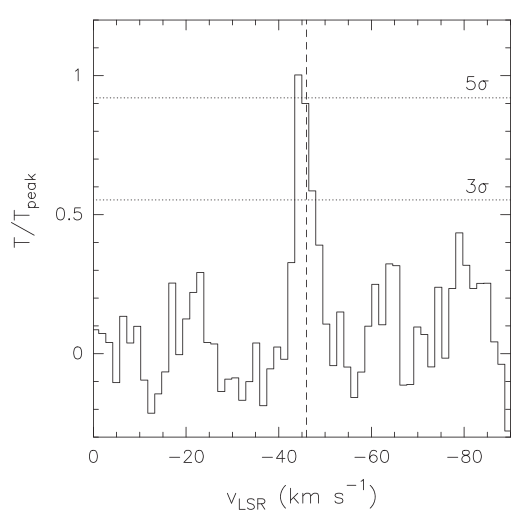

Figure 6. Composite normalized spectrum of PO toward $\mathrm{W} 3(\mathrm{OH})$. The dashed vertical line indicates the systemic velocity of the source, while the dotted horizontal lines show the $3 \sigma$ and $5 \sigma$ detection levels.

using the model based on Vasyunin \& Herbst (2013), which simulates the chemical evolution of a parcel of gas and dust with time-dependent physical conditions. First, the formation of a dark dense clump from a translucent cloud during free-fall collapse is simulated ("cold starless phase"). During this phase, the density increases from $10^{3}$ to $10^{6} \mathrm{~cm}^{-3}$ and visual extinction rises from $A_{\mathrm{v}}=2$ to $A_{\mathrm{v}} \gg 100 \mathrm{mag}$. Gas and dust temperatures are both decreasing slightly from 20 to $10 \mathrm{~K}$. On a second phase, the dense dark clump warms up from 10 to $200 \mathrm{~K}$ during $2 \times 10^{5} \mathrm{yr}$, thus developing into a hot core ("warm-up protostellar phase"). We have defined as time 0 the moment when the protostar starts to heat up the environment.

We used two sets of initial chemical abundances to explore the influence of the poorly constrained P depletion factor: (i) the "low metal" model (EA1, see Table 1 in Wakelam \& Herbst 2008), where the atomic P is depleted and has an abundance of $2 \times 10^{-10}$ with respect to hydrogen, typically used in dark cloud chemical models; and (ii) the "high metal" model, where the phosphorus abundance is increased by a factor of $\sim 25$ to $5 \times 10^{-9}$.

The results of the modeling are shown in Figure 7, which shows the temporal evolution of the temperature (Figure 7(a)), gas abundance of water (Figure 7(b)), gas abundances of PO 
and PN (Figure 7(c)), and the PO/PN ratio (Figure $7(\mathrm{~d})$ ). The two P-bearing species are chemically related and are formed purely in a sequence of gas-phase ion-molecule and neutralneutral reactions. PO is efficiently formed during the cold collapse phase in a sequence of reactions:

$$
\begin{gathered}
\mathrm{H}_{3} \mathrm{O}^{+}+\mathrm{P} \longrightarrow \mathrm{HPO}^{+}+\mathrm{H}_{2} \\
\mathrm{HPO}^{+}+\mathrm{e}^{-} \longrightarrow \mathrm{PH}+\mathrm{O} \\
\mathrm{HPO}^{+}+\mathrm{e}^{-} \longrightarrow \mathrm{PO}+\mathrm{H} \\
\mathrm{P}^{+}+\mathrm{H}_{2} \longrightarrow \mathrm{PH}_{2}^{+} \\
\mathrm{PH}_{2}^{+}+\mathrm{e}^{-} \longrightarrow \mathrm{PH}+\mathrm{H} \\
\mathrm{O}+\mathrm{PH} \longrightarrow \mathrm{PO}+\mathrm{H} .
\end{gathered}
$$

$\mathrm{PN}$ is mainly formed from $\mathrm{PO}$ via

$$
\mathrm{N}+\mathrm{PO} \longrightarrow \mathrm{PN}+\mathrm{O} \text {. }
$$

During the starless phase the model predicts that PO is more abundant than PN (Figure 7(c)). Both species freeze out to grains at the end of the cold collapse phase and consequently the gas abundances sharply decrease (Figure 7(c)). PO has an abundance $\sim 5$ times higher than $\mathrm{PN}$ at that time.

At the warm-up phase, once the temperature gradually increases from 10 to $200 \mathrm{~K}$ (Figure 7(a)), the species evaporate to the gas phase in the order determined by their desorption energies. PO and PN desorb simultaneously when the temperature reaches $\sim 35 \mathrm{~K}\left(\sim 0.7 \times 10^{5} \mathrm{yr}\right.$, Figure $\left.7(\mathrm{c})\right)$, because in our model they have the same evaporation energy of $1900 \mathrm{~K}^{12}$

Some PO is converted to PN via gas-phase reactions, and the abundances of the two species become almost equal until $\sim 1.2$ $\times 10^{5} \mathrm{yr}$ (Figure $7(\mathrm{c})$ ). Once the temperature reaches $\sim 100 \mathrm{~K}$, water ice evaporates and the gas-phase abundance of water reaches a high value of $10^{-4}$ (see Figure $7(\mathrm{~b})$ ). Then, the abundance of protonated water $\mathrm{H}_{3} \mathrm{O}^{+}$correspondingly increases, and $\mathrm{PN}$ reacts with $\mathrm{H}_{3} \mathrm{O}^{+}$:

$$
\mathrm{PN}+\mathrm{H}_{3} \mathrm{O}^{+} \longrightarrow \mathrm{HPN}^{+}+\mathrm{H}_{2} \mathrm{O} \text {. }
$$

In turn, $\mathrm{HPN}^{+}$has two equally probable channels of dissociative recombination:

$$
\begin{aligned}
& \mathrm{HPN}^{+}+\mathrm{e}^{-} \longrightarrow \mathrm{PN}+\mathrm{H} \\
& \mathrm{HPN}^{+}+\mathrm{e}^{-} \longrightarrow \mathrm{PH}+\mathrm{N} .
\end{aligned}
$$

Since the abundance of atomic oxygen is higher by almost an order of magnitude than the abundance of atomic nitrogen, $\mathrm{PH}$ is preferably converted to $\mathrm{PO}$ rather than PN. As such, PN is gradually destroyed, while PO is additionally produced, which significantly increases the PO/PN ratio (Figure $7(d)$ ).

To compare the results of the observations with the predictions of our chemical models, we show the values of the abundances and the $\mathrm{PO} / \mathrm{PN}$ ratio derived from the observations (Table 3) in Figures 7(c) and (d), respectively. The main source of uncertainty of the estimated abundances arises from the uncertainty of the size of the emitting region and the $\mathrm{H}_{2}$ column density. We have considered a conservative

\begin{tabular}{|c|c|c|c|c|}
\hline \multirow[t]{3}{*}{ Source } & $N_{\mathrm{PN}}$ & $N_{\mathrm{PO}}$ & \multirow[t]{3}{*}{$N_{\mathrm{PO}} / N_{\mathrm{PN}}$} & \multirow[t]{3}{*}{ References } \\
\hline & \multicolumn{2}{|c|}{ Star-forming Regions } & & \\
\hline & $\times 10^{11} \mathrm{~cm}^{-2}$ & $\times 10^{11} \mathrm{~cm}^{-2}$ & & \\
\hline W51 e1/e2 & 210 & 400 & 1.8 & (1) \\
\hline $\mathrm{W} 3(\mathrm{OH})$ & 20 & 60 & 3.0 & (1) \\
\hline AFGL5142-EC ${ }^{\mathrm{a}}$ & 8.3 & $<20$ & $<2.4$ & (2) \\
\hline $05358-\mathrm{mm}^{\mathrm{a}}$ & 2.9 & $<13$ & $<4.5$ & (2) \\
\hline AFGL5142-MM ${ }^{\mathrm{a}}$ & 6.9 & $<12$ & $<1.7$ & (2) \\
\hline $18089-1732^{\mathrm{a}}$ & 3.3 & $<6.3$ & $<1.9$ & (2) \\
\hline $18517-0437^{\mathrm{a}}$ & 2.0 & $<4.4$ & $<2.2$ & (2) \\
\hline G5.89-0.39 ${ }^{\mathrm{a}}$ & 10.4 & $<14$ & $<1.3$ & (2) \\
\hline $19410+2336^{\mathrm{a}}$ & 1.6 & $<3.3$ & $<2.0$ & (2) \\
\hline $\mathrm{ON} 1^{\mathrm{a}}$ & 3.8 & $<6.3$ & $<1.7$ & (2) \\
\hline \multicolumn{5}{|c|}{ Protostellar Shocks } \\
\hline \multirow[t]{3}{*}{$\mathrm{L} 1157 \mathrm{~B}^{\mathrm{a}}$} & $4.2-11$ & $<5.3$ & $<0.5-1.3$ & (3) \\
\hline & \multicolumn{2}{|c|}{ Envelopes of Evolved Stars } & & \\
\hline & $\times 10^{15} \mathrm{~cm}^{-2}$ & $\times 10^{15} \mathrm{~cm}^{-2}$ & & \\
\hline VY Canis Majoris & 1.2 & 2.8 & 2.2 & (4) \\
\hline IK Tauri & 2.2 & $0.4-4.4$ & $0.17-2$ & (5) \\
\hline
\end{tabular}
range of one order of magnitude uncertainty for the abundances, i.e., $(0.5-5) \times 10^{-10}$ (see Figure 7(c)). In the case of the $\mathrm{PO} / \mathrm{PN}$ ratio, which is independent of the $\mathrm{H}_{2}$ column density, we have considered a lower uncertainty of a

\footnotetext{
${ }^{12}$ To the best of our knowledge, there are no experimental data on $\mathrm{PN}$ and $\mathrm{PO}$ desorption energies and, therefore, the results of the modeling should be accepted with some caution.
}

Table 5

Column Densities and Relative Abundances of PO and PN in Different Sources

Note.

a Beam-averaged column densities.

References. (1) This paper; (2) Fontani et al. (2016); (3) Aota \& Aikawa (2012); (4) Tenenbaum et al. (2007); (5) De Beck et al. (2013).

factor of 5, i.e., values in the range $1-5$. These observational constraints can only be explained by the "high metal" model (Figure $7(\mathrm{c})$ ), and during a period of $\sim 5 \times 10^{4} \mathrm{yr}$ (between 0.8 and $1.2 \times 10^{5} \mathrm{yr}$ in Figure 7$)$. This suggests that the value of the initial depleted atomic abundance of $\mathrm{P}$ is a factor of $\sim 25$ higher (i.e., $5 \times 10^{-9}$ ) than that commonly assumed by the standard "low metal" model.

The temporal window that fulfills the observational constraints translates in a temperature range between the desorption temperature of the P-bearing molecules $(35 \mathrm{~K})$ and $\sim 90 \mathrm{~K}$ (see Figure 7(a)). The former temperature is the same as that derived from PN transitions toward W51, which may indicate that the observations have detected the bulk of PN (and PO) that have recently desorbed from dust grains. The latter temperature is just below the sublimation temperature of the bulk of water $(100 \mathrm{~K})$, when $\mathrm{PN}$ is efficiently destroyed and partially converted to PO. Then, the gas abundance of water predicted by the model at this stage is still low, $\sim 10^{-9}$, which is in good agreement with the values of $5 \times 10^{-10}-4 \times 10^{-8}$ found toward several massive star-forming regions by Marseille et al. (2010; see Figure 7(b)).

\section{SUMMARY AND CONCLUSIONS}

We report on the first detection of the key prebiotic molecule PO toward two star-forming regions: W51 e1/e2 and W3(OH). The derived molecular abundances of PO are $\sim 10^{-10}$ in both sources. We have found an abundance ratio $\mathrm{PO} / \mathrm{PN}$ of 1.8 and 3 for $\mathrm{W} 51 \mathrm{e} 1 / \mathrm{e} 2$ and $\mathrm{W} 3(\mathrm{OH})$, respectively, in agreement with the values estimated for evolved stars. Our chemical modeling indicates that the two molecules are chemically related and are formed via gas-phase ion-molecular and neutral-neutral reactions during the cold collapse. The molecules freeze out 


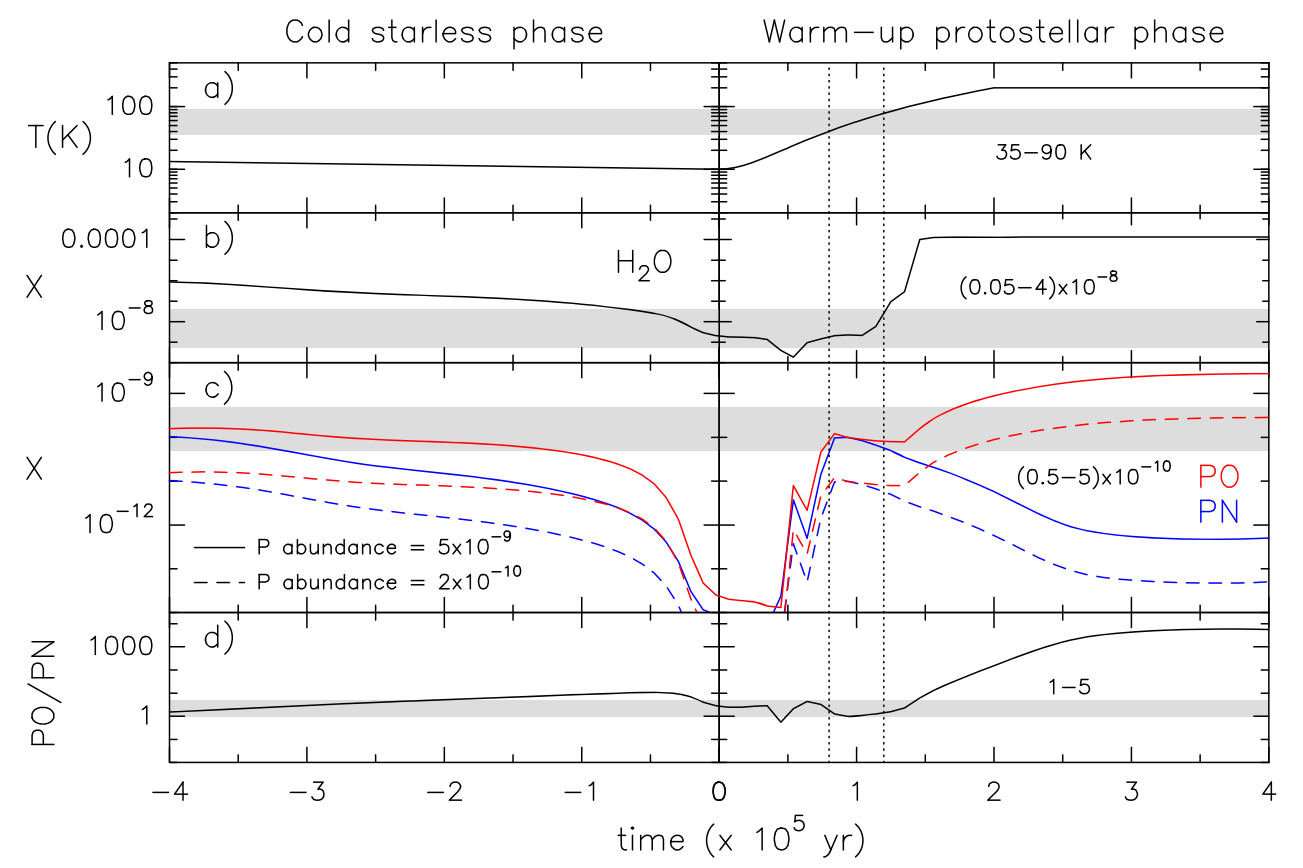

Figure 7. Results of our chemical model. The left panels correspond to the cold collapse phase, and the right panels to the subsequent warm-up phase. We show the evolution during $0.8 \times 10^{5} \mathrm{yr}$, time 0 being the moment when the protostar starts to heat up the environment. The two vertical dotted lines in the right panels indicate the temporal range for which the observational constraints are fulfilled (see text). (a) Evolution of the temperature assumed by the model. The gray band corresponds to the temperature range between 35 and $90 \mathrm{~K}$ which is compatible with the observational constraints (see text). (b) $\mathrm{H}_{2} \mathrm{O}$ abundance. The gray band corresponds to the range found toward massive star-forming regions by Marseille et al. (2010). (c) PO and PN abundances predicted by the model (red and blue lines, respectively). We have considered two different initial abundances of depleted atomic phosphorus: $5 \times 10^{-9}$ (solid lines, "high metal" model) and $2 \times 10^{-10}$ (dashed lines, "low metal" model, usually assumed in dark cloud models). The gray band indicates a conservative range of one order of magnitude around the molecular abundances derived from our observations of PO and PN (see text). (d) Ratio of the molecular abundances of PO and PN. The gray band shows a conservative range (1-5) around the ratio found by the observations.

onto grains at the end of the collapse, and evaporate during the warm-up phase once the temperature reaches $\sim 35 \mathrm{~K}$. Similar abundances of $\mathrm{PO}$ and $\mathrm{PN}$ are expected during a period of $\sim 5$ $\times 10^{4} \mathrm{yr}$ at the early stages of the warm-up phase, when the temperature is in the range $35-90 \mathrm{~K}$. The observed molecular abundances require a relatively high initial abundance of depleted atomic phosphorus of $5 \times 10^{-9}, 25$ times higher than the "low metal" P-abundance typically used in dark cloud chemical models.

The authors are grateful to the staff of IRAM for their help during the observations of the IRAM $30 \mathrm{~m}$ data, and in particular to the Director for allowing us to use the telescope during the DDT proposal D04-15. This work was partly supported by the Italian Ministero dell'Istruzione, Università e Ricerca through the grant Progetti Premiali 2012-iALMA. P.C. and A.V. acknowledge the financial support of the European Research Council (ERC; project PALs 320620). J.M.-P. acknowleges partial support from the MINECO under grants AYA2010-2169-C04-01, FIS2012-39162-C06-01, ESP201347809-C03-01, and ESP2015-65597-C4-1.

\section{APPENDIX \\ CALCULATION OF THE HYDROGEN COLUMN DENSITY}

One can derive the hydrogen column density of a clump from the continuum flux emitted by the dust. Assuming an homogeneous clump with temperature $T_{d}$ and opacity $\tau_{\nu}$ at frequency $\nu$, the solution of the radiative transfer equation gives the intensity emitted:

$$
I_{\nu}=B_{\nu}\left(T_{d}\right)\left(1-e^{-\tau_{\nu}}\right),
$$

where $B_{\nu}$ is the Planck function:

$$
B_{\nu}=\frac{2 h \nu^{3}}{c^{2}} \frac{1}{e^{h \nu / k T_{\mathrm{d}}}-1},
$$

where $c$ is the speed of light, $k$ is the Boltzmann constant, and $h$ is the Planck constant.

One can relate the flux density measured within the beam solid angle of the telescope $(\Omega)$ with the intensity using

$$
I_{\nu}=\frac{F_{\nu}}{\Omega} .
$$

Combining Equations (12) and (14), one can derive the opacity of the dust emission inside the beam:

$$
\tau_{\nu}=-\ln \left(1-\frac{F_{\nu}}{\Omega B_{\nu}\left(T_{d}\right)}\right) .
$$

Since the opacity is defined as

$$
\tau_{\nu}=\int \kappa_{\nu} \rho d r
$$

where $\rho$ is the volume density and $\kappa_{\nu}$ is the absorption coefficient per unit density, one can derive the hydrogen column density with the optical depth using

$$
N_{\mathrm{H}_{2}}=\int \frac{\rho}{\mu m_{\mathrm{H}}} d r=\frac{\tau_{\nu}}{\mu m_{\mathrm{H}} \kappa_{\nu}},
$$

where $m_{\mathrm{H}}$ is the hydrogen mass and $\mu=2.8$ is the mean molecular mass of the ISM with respect to $\mathrm{H}_{2}$ molecules. We 
Table 6

Continuum Fluxes of W51 e1/e2 and W3(OH) Clumps from ATLASGAL and SCUBA, Respectively, and Derived Dust Opacities and Molecular Hydrogen Column Densities

\begin{tabular}{lcccc}
\hline \hline Source & $\lambda(\mu \mathrm{m})$ & $F_{\nu}\left(\mathrm{Jy} \mathrm{beam}^{-1}\right)$ & $\tau$ & $N_{\mathrm{H}_{2}}\left(\times 10^{23} \mathrm{~cm}^{-2}\right)$ \\
\hline W51 & 870 & $70.2^{\mathrm{a}}$ & 0.02 & 2.0 \\
W3(OH) & 850 & $26.3^{\mathrm{b}}$ & 0.005 & 0.5 \\
\hline
\end{tabular}

Notes.

${ }^{a}$ From ATLASGAL (Csengeri et al. 2014).

${ }^{\text {b }}$ From SCUBA (Di Francesco et al. 2008).

have used Equations (15) and (17) to derive the dust opacity and the hydrogen column density of W51 and W3(OH) from the dust continuum emission from ATLASGAL $(870 \mu \mathrm{m}$; Csengeri et al. 2014) and SCUBA $(850 \mu \mathrm{m}$; Di Francesco et al. 2008) surveys, respectively. We have considered that the dust temperature is the same as the excitation temperature derived for PN, $35 \mathrm{~K}$. We have adopted a gas-to-dust ratio of 100 and $\kappa_{\nu}=1.85 \mathrm{~cm}^{2} \mathrm{~g}^{-1}$, from interpolation of column 5 of Table 1 of Ossenkopf \& Henning (1994). We have used the peak flux densities shown in Table 6, and the corresponding telescope beams: 19!"2 (ATLASGAL) and 22!"9 (SCUBA). The results are given in Table 6 . We have used the values of $N_{\mathrm{H}_{2}}$ to compute the molecular abundances of PN and PO (see Table 3).

\section{REFERENCES}

Adams, N. G., McIntosh, B. J., \& Smith, D. 1990, A\&A, 232, 443 Agúndez, M., Cernicharo, J., Decin, L., Encrenaz, P., \& Teyssier, D. 2014, ApJL, 790, L27

Aota, T., \& Aikawa, Y. 2012, ApJ, 761, 74

Caffau, E., Andrievsky, S., Korotin, S., et al. 2016, A\&A, 585, A16

Caffau, E., Bonifacio, P., Faraggiana, R., \& Steffen, M. 2011, A\&A, 532, A98

Charnley, S. B., \& Millar, T. J. 1994, MNRAS, 270, 570

Csengeri, T., Urquhart, J. S., Schuller, F., et al. 2014, A\&A, 565, A75

De Beck, E., Kamiński, T., Patel, N. A., et al. 2013, A\&A, 558, A132

Demyk, K., Wlodarczak, G., \& Carvajal, M. 2008, A\&A, 489, 589
Di Francesco, J., Johnstone, D., Kirk, H., MacKenzie, T., \& Ledwosinska, E. 2008, ApJS, 175, 277

Fontani, F., Rivilla, V. M., Caselli, P., Vasyunin, A., \& Palau, A. 2016, ApJ, 822,30

Grevesse, N., \& Sauval, A. J. 1998, SSRv, 85, 161

Hachisuka, K., Brunthaler, A., Menten, K. M., et al. 2006, ApJ, 645, 337

Ikeda, M., Ohishi, M., Nummelin, A., et al. 2001, ApJ, 560, 792

Jura, M., \& York, D. G. 1978, ApJ, 219, 861

Kalenskii, S. V., \& Johansson, L. E. B. 2010, ARep, 54, 1084

Kanata, H., Yamamoto, S., \& Saito, S. 1988, JMoSp, 131, 89

Kawaguchi, K., Saito, S., \& Hirota, E. 1983, JChPh, 79, 629

Koo, B.-C., Lee, Y.-H., Moon, D.-S., Yoon, S.-C., \& Raymond, J. C. 2013, Sci, 342, 1346

Liu, S.-Y., Mehringer, D. M., \& Snyder, L. E. 2001, ApJ, 552, 654

Lykke, J. M., Favre, C., Bergin, E. A., \& Jørgensen, J. K. 2015, A\&A, 582, A64

Maciá, E., Hernández, M. V., \& Oró, J. 1997, OrLi, 27, 459

Marseille, M. G., van der Tak, F. F. S., Herpin, F., et al. 2010, A\&A, 521, L32

Martín-Pintado, J., Fontani, F., Caselli, P., \& Cesaroni, R. 2016, A\&A, submitted

Matthews, H. E., Feldman, P. A., \& Bernath, P. F. 1987, ApJ, 312, 358

Millar, T. J., Bennett, A., \& Herbst, E. 1987, MNRAS, 229, 41

Ossenkopf, V., \& Henning, T. 1994, A\&A, 291, 943

Pasek, M. A., \& Lauretta, D. S. 2005, AsBio, 5, 515

Qin, S.-L., Schilke, P., Wu, J., et al. 2015, ApJ, 803, 39

Remijan, A., Snyder, L. E., Liu, S.-Y., Mehringer, D., \& Kuan, Y.-J. 2002, ApJ, 576, 264

Rivilla, V. M., Fontani, F., Beltran, M. T., et al. 2016, arXiv:1605.06109

Roederer, I. U., Jacobson, H. R., Thanathibodee, T., Frebel, A., \& Toller, E. 2014, ApJ, 797, 69

Scott, P. F. 1978, MNRAS, 183, 435

Sutton, E. C., Blake, G. A., Masson, C. R., \& Phillips, T. G. 1985, ApJS, 58,341

Tenenbaum, E. D., Woolf, N. J., \& Ziurys, L. M. 2007, ApJL, 666, L29

Thorne, L. R., Anicich, V. G., Prasad, S. S., \& Huntress, W. T., Jr. 1984, ApJ, 280, 139

Turner, B. E., \& Bally, J. 1987, ApJL, 321, L75

Vasyunin, A. I., \& Herbst, E. 2013, ApJ, 762, 86

Wakelam, V., \& Herbst, E. 2008, ApJ, 680, 371

Wilner, D. J., Welch, W. J., \& Forster, J. R. 1995, ApJL, 449, L73

Wyrowski, F., Schilke, P., Walmsley, C. M., \& Menten, K. M. 1999, ApJL, 514, L43

Xu, Y., Reid, M. J., Menten, K. M., et al. 2009, ApJ, 693, 413

Zapata, L. A., Rodríguez-Garza, C., Rodríguez, L. F., Girart, J. M., \& Chen, H.-R. 2011, ApJL, 740, L19

Zhang, Q., \& Ho, P. T. P. 1997, ApJ, 488, 241

Zhang, Q., Ho, P. T. P., \& Ohashi, N. 1998, ApJ, 494, 636

Ziurys, L. M., \& Friberg, P. 1987, ApJL, 314, L49 